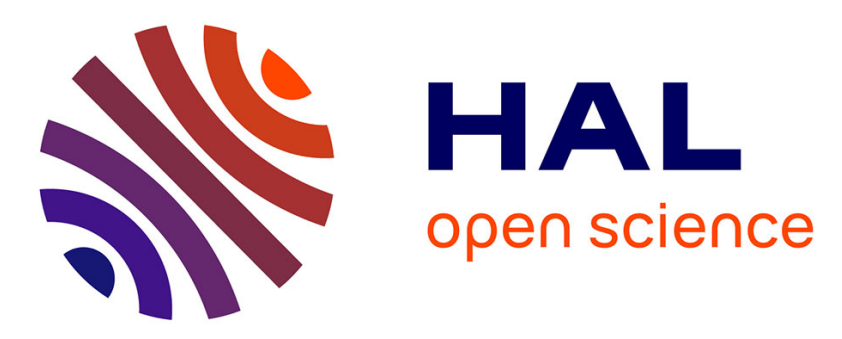

\title{
A New Relational Spatial OLAP Approach For Multi-resolution and Spatio-multidimensional Analysis of Incomplete Field Data
}

Mehdi Zaamoune, Sandro Bimonte, François Pinet, Philippe Beaune

\section{- To cite this version:}

Mehdi Zaamoune, Sandro Bimonte, François Pinet, Philippe Beaune. A New Relational Spatial OLAP Approach For Multi-resolution and Spatio-multidimensional Analysis of Incomplete Field Data. ICEIS 2013 INSTICC International Conference on Enterprise Information Systems, Jul 2013, Angers, France. p. hal-00965471

\section{HAL Id: hal-00965471 \\ https://hal.uca.fr/hal-00965471}

Submitted on 25 Mar 2014

HAL is a multi-disciplinary open access archive for the deposit and dissemination of scientific research documents, whether they are published or not. The documents may come from teaching and research institutions in France or abroad, or from public or private research centers.
L'archive ouverte pluridisciplinaire HAL, est destinée au dépôt et à la diffusion de documents scientifiques de niveau recherche, publiés ou non, émanant des établissements d'enseignement et de recherche français ou étrangers, des laboratoires publics ou privés. 


\title{
A New Relational Spatial OLAP Approach For Multi-resolution and Spatio-multidimensional Analysis of Incomplete Field Data
}

\author{
Mehdi Zaamoune ${ }^{1}$, Sandro Bimonte ${ }^{1}$, François Pinet $^{1}$, Philippe Beaune ${ }^{2}$ \\ ${ }^{I}$ IRSTEA, TSCF, 24 Av. des Landais, Aubière, France \\ ${ }^{2}$ Agaetis, 10 allée Evariste Galois, 63000 Clermont-Ferrand \\ \{mehdi.zaamoune,sandro.bimonte,francois.pinet\}@irstea.fr, pbeaune@agaetis.fr
}

\begin{abstract}
Keywords: Spatial OLAP, incomplete continuous field, multi-resolution geographic data
Abstract: Integrating continuous spatial data into SOLAP systems is a new research challenge. Moreover, representation of field data at different scales or resolutions is often mandatory for an effective analysis. Thus, in this paper, we propose a logical model to integrate spatial dimensions representing incomplete field data at different resolutions in a classical SOLAP architecture.
\end{abstract}

\section{INTRODUCTION}

Spatial Data Warehouse (SDW) and Spatial OLAP (SOLAP) systems play an important role in helping decision-makers obtain the maximum benefits of these large amounts of geographic data (Bédard, Merrett et al. 2001). These technologies extend Data Warehouse (DW) and OLAP systems to integrate spatial data with warehoused classical data to achieve the on-line analysis of large georeferenced data sets. SOLAP systems integrate advanced OLAP and Geographic Information Systems (GIS) in a unique framework usually based on the relational storage (i.e. Oracle, etc.) of spatial data according to the vector model, and their analysis through SOLAP operators (Spatial Roll-Up, Spatial Slice, etc.) implemented by the SOLAP server (e.g. Map4Decision, etc.) and visualized by means of tabular, graphical and cartographic displays (Gomez, Gomez et al. 2012). SDW are modeled according to the spatio-multidimensional model that extends the traditional multidimensional model to define spatial dimensions (i.e. analysis axes with spatial attributes) and spatial measures (i.e. analysis subjects) that integrate geographic information using the vector model (Bédard, Rivest et al. 2007). SOLAP technology can be applied in different domains (e.g. archeology, public health, etc.).

Geographic information can be represented by two models, depending on the nature of data: discrete (vector) and continuous field (Mennis, Viger et al. 2005). Continuous fields (also called continuous spatial data) represent physical phenomena that continuously change in space (Paolino, Sebillo et al. 2010), for example the temperature, population, etc. Two representations of field data have been proposed: incomplete and complete (Paolino, Sebillo et al. 2010). Incomplete representations store a sample of points and need additional functions to calculate the field in nonsampled areas (e.g. grid of points, TIN, etc.) (e.g. Figure 2). Complete representations associate estimated values to regions and assume that these values are valid for each point in the regions (e.g. raster). For those representations some ad-hoc analysis operators have been defined that allow a point by point analysis (i.e. map algebra (Mennis, Viger et al. 2005)). Representation of geographic data at different scales or resolutions (e.g. Figure 2b) is mandatory for an effective analysis of spatial complex phenomena since it represents a geovisualization method (Camossi, Bertino et al. 2009). Consequently, these resolutions or scales represent decision-makers analysis needs that should be explicitly represented in any data and query model. Indeed, in the context of Geographic Information Systems and Spatial Databases Management Systems (SDBSM), several works addresses this issue by proposing conceptual, logical and physical data models and analysis techniques (Parent, spaccapietra et al. 2006).

Motivated by the important analysis capabilities offered by the continuous field representation of geographic data when integrated in SOLAP systems 
(visualization, querying, etc.) recently some works investigated the extension of the spatiomultidimensional model and SOLAP operators with complete and incomplete field data (cf. Section 6). In the same way, handling multi-resolutions of spatial data into spatial multidimensional models has been proposed in few works (Yvan, Proulx et al. 2002) (Gascueña and Guadalupe 2009) that propose conceptual models to represent SDW with several representations (scales, resolutions, etc.) of spatial dimensions and measures.

However, to best of our knowledge existing works concerning field data and multi-resolutions lack of a complete implementation in a full-featured SOLAP architecture, or in other terms they do not propose a coupled relational and SOLAP server model for a generic SOLAP architecture allowing (i) the map algebra operators, (ii) the multi-resolution, and (iii) a continuous view of the field.

In order to handle the spatio-multidimensional analysis of incomplete regular grid field data at different resolutions, we propose in this paper: (i) a specific logical model, extending the well-know relational star schema; (ii) and some new MDXbased defined functions. We validate our proposal using a real case study concerning the odor monitoring, and we provide some experiments showing the feasibility also in terms of storage and computation performances.

\section{MODELING AND ANALYSIS REQUIREMENTS}

In order to show our proposal, we present a case study based on data issued from the monitoring of urban odor. For each 15 minutes and type of odor (e.g. NO2) a regular grid map (field) is produced by means of some sample points and a simulation model (ADMS5). The simulation model estimates odors for a whole urban area and produces $100 * 100$ thematic grids. Examples of points grid are provided in figure 2-a (odor values are represented by color: green, yellow, red) for 10:00 19-2-2012 and 10:15 19-2-2012. Let us now suppose that the user wants to aggregate data along a temporal dimension (year, month, day, hour, minute) using the average to obtain an aggregated odor map. This is an OLAP operation of RollUp on the temporal dimension that corresponds to a local map algebra operation (Figure 2 -a). Moreover, since space is represented in a continuous way, decision-makers should be able to ask for the result of any OLAP query in any point of the spatial dimension (for example, s/he should be interested in the odor value at 10:00 in the area behind the building) (Figure 2-c). It is also possible to apply a spatial slice operator on the spatial dimension (i.e. using a spatial predicate to select a subset of warehoused data) (Figure 2-d). In order to answer to these last two queries spatial interpolation methods are necessary, since in incomplete field only the values provided by the simulation model are stored. Spatial interpolation is the process of prediction of almost exact values of attributes at unsampled locations from measurements made at control points within the same area (O'Sullivan and J.Unwin 2002). In our case the interpolation function used is the bilinear interpolation, which is a local deterministic method. It uses the $2 * 2$ grid sample points closest to the unknown point and calculates a distance weighted average which determines in what proportion the value of a neighbour impact on the value of the point to be estimated (Figure 1).

Finally, as stated in the previous section, since visualization of spatial data at different resolutions is mandatory for the exploration/analysis process, decision-makers should be able querying spatial warehoused data at different resolutions. It is very important to note that for each spatial phenomenon a set of useful known resolutions exist, so they could be predefined according to data and users needs. Moreover, in order to calculate values at finer resolutions spatial interpolation functions as previously described can be used.

To summarize, spatio-multidimensional analysis of field data implies: supporting (i) OLAP classical operators as Map Algebra, (ii) continuous view of spatial data, (iii) spatial slice operators using field data, and (iv) visualizing and querying data at different predefined resolutions.

\section{Spatio-multidimensional model for incomplete field data}

In this section we describe our spatiomultidimensional model for handling incomplete fields at different resolutions. Our model extends the classical spatio-multidimensional models to generate the continuity of the phenomena over the studied area, and represents pre-defined levels of resolution. In particular, a "Cube" is composed of "Facts" and "Dimensions". A "Dimension" is composed of "Hierarchies", which are composed of "Levels". A "Level" can be spatial or conventional. This means that it can contain "Spatial attributes" (e.g. points, etc.), or contain only alphanumerical attributes respectively. "Facts" is composed of "Spatial Measures" or "Conventional Measures". Moreover, our extension defines a "Field level" as a special type of spatial level where each member has a geometric attribute (e.g. point), a "neighbourhood 
relationship" association, and a resolution level to which it belongs.

(a)
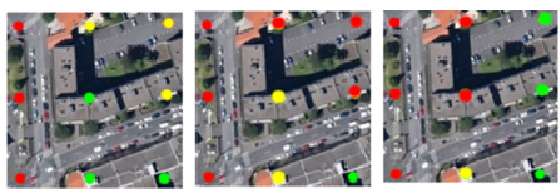

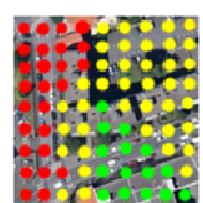

10:00

(b)

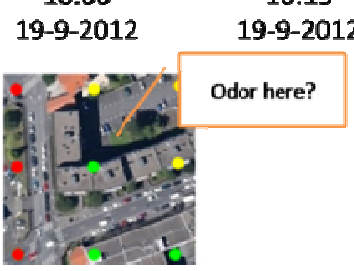

(c)

10:00

19-9-2012

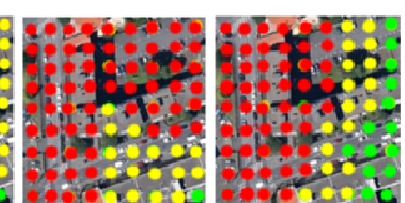

10 Hour $19-9-2012$

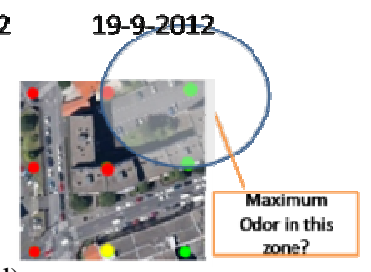

(d)

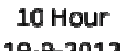

Figure 2: a) local map algebra aggregation over incomplete field. b) multiresolution representation over incomplete field c) querying an unsampled point d) querying an unsampled zone

Indeed, as described in the previous section a spatial interpolation function uses a set of points, which depend on the function (e.g. bilinear function uses a $2 * 2$ grid), to estimate the unknown value. Thus, in our approach values are represented by measures, the "Neighbourhood relation" links each detailed "Field level" to its neighbours in the less detailed level. As soon as the value of a high resolution "Field level" is required its neighbours are found through the "Neighbourhood relation". However, this type of relationship can be implemented in different ways, depending on the intended purpose as shown in the next section. In our case, we want to estimate the value of any point (x, y) of a Field level, so neighbours are found on the fly by the relation "Neighbourhood relation". In the case of a change in the level of resolution, the members of a high resolution (e.g. 200*200), are predefined and therefore, their respective neighbours, which belongs to the resolution $100 * 100$, can be pre-stored as attributes.

As described in the model, a hierarchy can contain several field levels representing the phenomena at different resolutions. This means that changing resolutions implies navigating into the hierarchy and calculating values by means of the interpolation function or an ad-hoc aggregation function when we move from less detailed resolution to more detailed one, or vice versa.

The odor SDW of our case study using our spatiomultidimensional model is shown in Figure 3.

This instance describes the dimensions and facts that constitute our cube.

In addition to the dimensions (Source, Tracer and Time), the "Facts" class has a classical measure "odorMeasure" and a derived measure "EstimatedOdorMeasure". The derived measure is calculated according to two functions:

a)"Interpolatepoint"(continuity).b)"InterpolateBiline ar" (multiresolution). In our case, the interpolation function used is the "bilinear interpolation". The relationship whose cardinalities are " 2,4 " represents the "Neighbourhood relation". A member of the "Incomplete Field Level" can have 2 or 4 neighbours, depending on its position in the grid $2 * 2$ that surrounds it. The "Neighbourhood relation" can be used to retrieve neighbours of a location $(\mathrm{x}, \mathrm{y})$ to estimate the value in that position (continuity), or to retrieve a high resolution member's neighbours in the lower level of resolution to estimate its value (multiresolution on the fly).

\section{$4 \quad$ Relational and OLAP models}

In this section we present the implementation of our spatio-multidimensional model in a typical relational SOLAP architecture based on SQL (the Relational DBMS standard language) and MDX, which is the de-facto standard of OLAP Servers. This provides a generality character to our approach, being possible to be implemented in any architecture of this kind.

Let us suppose to have one "Field level" representing points at the resolution $100 * 100$, and then the logical model of our case study is represented as in fig. 4. It is a classical star schema. This model is composed of a fact table containing measures with foreign keys to dimension tables. Each dimension table is denormalized, and has attributes representing levels.

Let us also suppose to have a classical OLAP model based on that logical schema, where the spatial level is called [Field]. [res100]. As we can see, the dimensions that constitute the model are: the temporal dimension, which consists of five levels of granularity (Year, month, day, hour and minute); the Source dimension that expresses the source of the pollutant (e.g. cars); the Tracer dimension is the type of pollutant (eg NO2), which is also defined by its identifier and name; and the "Field dimension" that represent a regular grid of points and consists of one level representing the regular grid at the $100 * 100$ resolution, which is 
composed of an identifier and a geometry representing a point. The measure «Concentration of odor» represents the values for all members representing the field at a $100 * 100$ resolution. This representation of incomplete field data in the multidimensional model allows making queries as Map Algebra operators (point by point aggregation) such as the following:

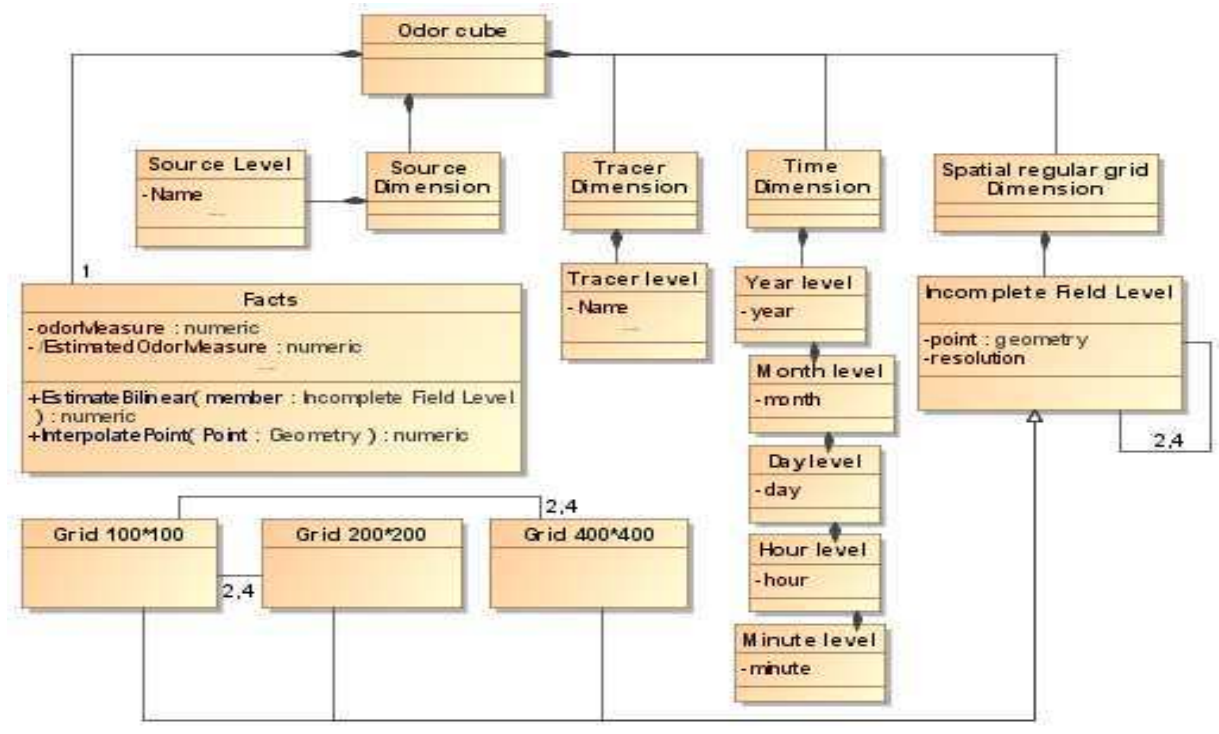

Figure 3: Odor SDW multi-dimensional model

Query 1: select average odor for each field member during 2012

SELECT [Field].[res100]. Members ON ROWS, \{[time].[2012]\} ON COLUMNS

FROM [odorCube] WHERE

[Measures].[value]

\subsection{Incomplete field}

In order to implement field levels we have defined a GeoMDX user-defined function that represents a spatial interpolation as:

NumericType InterpolatePoint (Geometry)

This function takes as input a geometry (point) and returns a numerical value, which is a derived measure in the OLAP model, representing an estimated value calculated using the neighbourhood values of the point given in input. Thus, let us suppose that we want to retrieve a value of the field in a location whose geometric property is set to the geometric coordinates POINT(-72.1235 42.3521). Then in order to answer to that need using the Bilinear interpolation function, decision-makers have to simply use a GeoMDX function in the following way: InterpolatePoint(POINT(72.123542 .3521 )).

Thus, the function will look for the neighbours of the point given as a parameter, in the field level ([Field].[res100]), on the basis of the distance, and then find neighbours' respective values in the fact table, evaluate the value of the point to estimate using these values, and then return an estimated derived measure. Here is an example query that uses the "InterpolatePoint" function:

Query 2: select a field member's value at coordinates (721148 3140020) for the year 2012.

With member [Measures]. [value] as'

InterpolatePoint (ST_GeomFromText ("

POINT (721148 3140020)"))'

SELECT [Measures]. [value] ON ROWS,

[time].[2012] ON COLUMNS

FROM [odorCube]

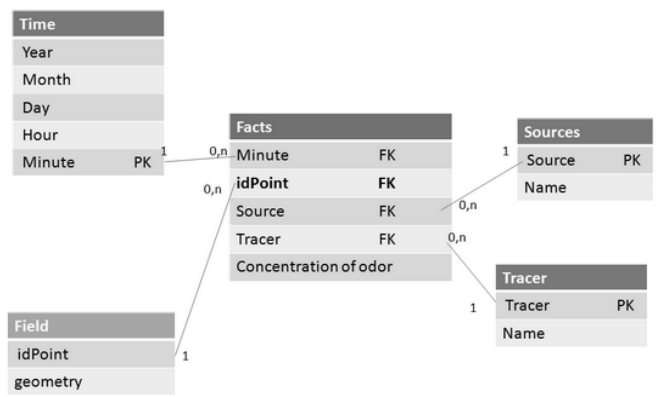

Figure 4: Classical star schema integrating regular grid of points

Note that generally MDX allows defining userdefined functions in several programming languages (i.e. Java, .NET, etc.) depending of the OLAP Server used. In this work we have used a Java-based 
implementation in GeoMondrian (see Sec. 5). In particular, the interpolation is done using an existing interpolation Java API "javax.media.jai api” (JAI).

In this way we achieve the continuous view of field data using incomplete fields as stated in Section 2.

\subsection{Multiresolution}

Theoretically, we can measure a value of a field at every position inside a geographic space. However, not all resolutions are necessarily relevant. Indeed, according to the type of analysis performed by the user, a more or less detailed resolution can be requested. The multiresolution is an approach that consists in defining resolution levels likely to improve the rendering of the requests made by the user. To model an incomplete field at several resolutions in a multi-dimensional model, we propose two Approaches based on the "Classical Star Schema": The "field aggregation star-schema" approach and the "field interpolation star schema" approach.

\subsubsection{Field Aggregation Star-Schema Approach}

Based on the star schema model previously described, we propose a logical schema where the spatial dimension presents different field levels at different resolutions (fig. 5-a). This model extends the spatial dimension of figure 4 with 2 other levels each representing a different level of resolution ([Field].[res200] and [Field].[res400]). Each level of the field dimension is composed of an identifier and a geometry representing a point. The fact table is associated, classically, to the most detailed level of the field dimension.

In this way, decision-maker can explore warehoused field data at different resolutions during the same analysis MDX-based session. Only need to change the level of resolution in the query to change the level of details of the result. Using this approach, we use in an MDX query, the appropriate level of resolution of the field dimension as in the following where the Query 1 becomes:

Query 3: select average odor for each field member at the 400*400 resolution during 2012

SELECT [Field]. [res400]. Members ON ROWS, \{[time].[2012]\} ON COLUMNSFROM [odorCube] WHERE [Measures]. [value]

\subsubsection{Field Interpolation Star-Schema Approach}

As stated in Section 2, in order to provide field data at finer resolutions, spatial interpolation methods can be used. Then, here we propose a variation of the previously proposed schema for handling multiple field resolution levels, by associating the fact table to the field at less detailed resolution as shown on figure 5-b. In our approach moving from fact table values to finer spatial members' values implies applying spatial interpolation functions. Note that this approach is possible only when dealing with spatial data, because according to the Tobler law geographical position of data can be used for estimating missing values.

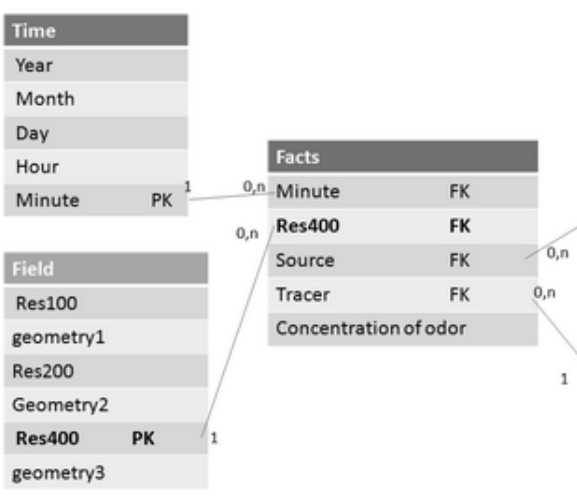

(a)

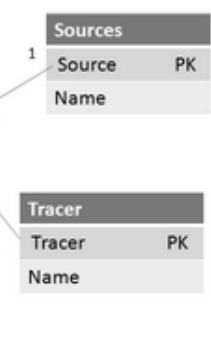

Figure 5: (a) Field Aggregation Star Schema (FASS), (b) Field Interpolation Star Schema (FISS) 
We have implemented a GeoMDX function in the same way of the function defined in Section 4.1:

Numeric-type InterpolateBilinear (Field Member)

However, this function, named "InterpolateBilinear" is prepared to receive as input a field level member instead of geometry and return an interpolated value of this member. We can also see that in this case, the neighbors of each member of a higher resolution than the original one are also stored in the "Field" (Neighbours2, Neighbours3), since members of each resolution are pre-defined in advance, but their values are not since they depend on other dimensions.

Calling this function as follows:

InterpolateBilinear

([Field].[res400].CurrentMember) in the formula of a derived measure, allows to find the values of all the members of the level "res400" (incomplete field at a $400 * 400$ resolution) using their neighbors "Neighbors3". Thus, the query 3 can be performed as follows:

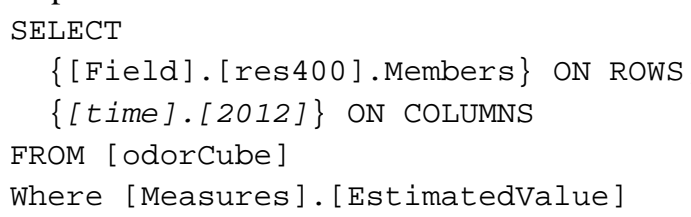

While in the multidimensional SOLAP schema, the "InterpolateBilinear" function is called in the "EstimatedValue" calculated measure formula as: formula=" InterpolateBilinear ([Field] . [ res400].CurrentMember)"

As we can see in the previous query, the call of the calculated measure enables to find the values at a given scale transparently to the decision maker as a classical aggregation (SQL). This approach is motivated by performance issues as described in the next section.

\section{Experimentations}

In this section we detail the performances of the two approaches proposed in Section 4.2 (FASS and FISS) in terms of storage and time computation. The computer used for the following tests has the following configuration: processor Intel ${ }^{\circledR}$ core ${ }^{\mathrm{TM}} \mathrm{i} 3$ 2,20 GHz, RAM 4 Go, Operating system Windows 7 professional, System OS 64 bits.

In particular, spatial data is stored in PostGIS Spatial DBMS. PostGIS is an open source software that adds support for geographic objects to the PostgreSQL object-relational database. PostGIS follows the Simple Features for SQL specification from the Open Geospatial Consortium (OGC); we use GeoMondrian as a SOLAP server; and JPivot as a client. GeoMondrian is an Open Source Spatial Online Analytical Processing Server.

In order to test our proposal we define different cases where the spatial dimension presents: one field level at the $100 * 100$ resolution; two levels at the resolutions $100 * 100$ and $200 * 200$; and finally three levels at the resolutions 100*100, 200*200 and $400 * 400$. We also vary the size of the temporal dimension in order to understand impact of the spatial and non spatial dimension on performances.

Figure 6-a shows the size of the fact table measured in function of the number of spatial and temporal members (spatial finest resolution / temporal finest granularity) using the two approaches. We can easily see two important differences: i) the field aggregation approach is expensive in terms of storage than the field interpolation one since the latter stores only facts values at a less detailed spatial granularity, ii) in the field interpolation approach the size of the fact table only varies depending on the size of the non spatial dimensions. Thus, even increasing the size of the spatial dimension, the fact table does not change since it contains only measures related to the first level of resolution.

In order to evaluate computation performance we execute the queries previously cited, where we combine roll-up operation on non spatial dimensions, and spatial slice operators over different field resolutions.

Figure 6-b represents the execution time of the query 3 , which consists in generating values of the members at different resolutions taking into account different sizes of the time dimension. This figure shows a certain degree of approximation in execution time between the two approaches to a certain level. Beyond this level, we note that the gap widens considerably. Thus, minimizing storage and relations has allowed the field interpolation approach we propose to have better execution time than the field aggregation approach at all resolution levels $(100 * 100,200 * 200$ and $400 * 400)$. Figure 9 shows that the execution time in the "field aggregation approach" increases depending on the number of spatial and temporal members, whereas in the "field interpolation approach", it increases mainly depending on the number of temporal members. Indeed the size of the spatial dimension does not influence much on performance, since there is no relationship between the fact table and the members who belong to high resolutions. 


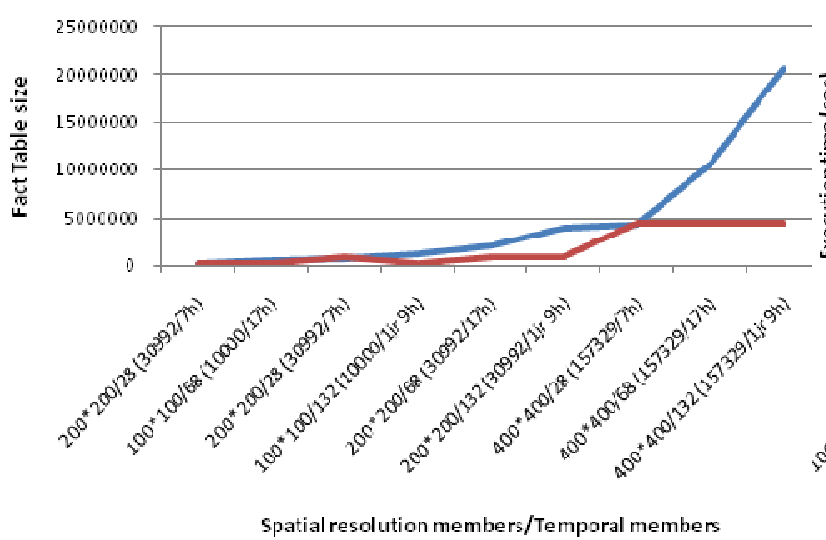

a)

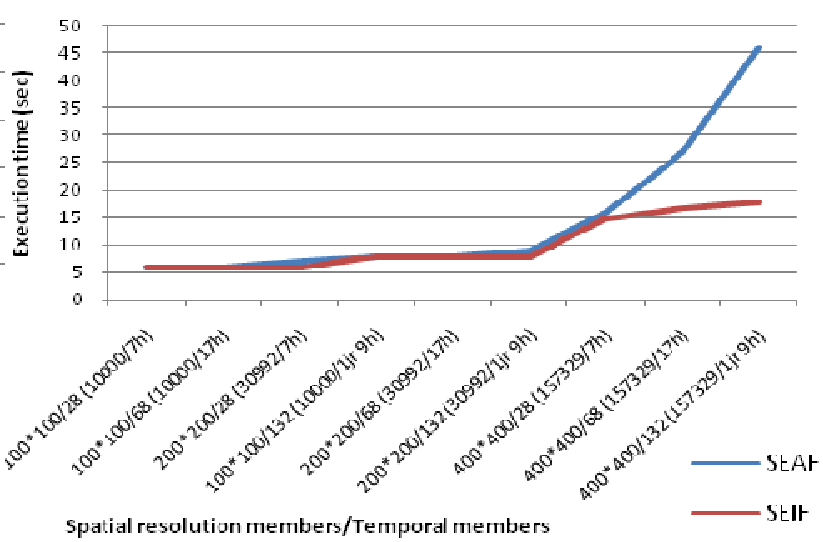

b)

Figure 6: a) Fact table size with the field aggregation and the field interpolation approaches b) Execution time for Query 3 for the Field aggregation Star Schema and the Field Interpolation Star Schema approaches

\section{Related work}

In order to integrate fields data in a SOLAP model, (Ahmed and Miquel 2005) propose a multidimensional model for handling continuous discrete fields, storing a sample of points as spatial members, to create a discrete cube which is interpolated in the client-side to simulate a continuity. (McHugh 2008) defines new types of dimensions handling fields as a regular grid of squares (raster): "hybrid dimension", "mixed hybrid dimension", "mixed matrix dimension" and "geometric matrix dimension". She also defines the "matrix cube" where facts are cells of the matrix grid. The "field aggregation approach" presented in section 4.2.1 is based on this work. However, the "field interpolation approach" we propose, although it gives the same result, it is more efficient in terms of storage and execution time. (Gomez, Gomez et al. 2012) presents a discrete data model for representing continuous fields and an algebra that makes use of OLAP operators (e.g. Dice, Slice, Roll-up, Drilldown ...). However, the discrete model the authors propose does not support the continuous aspect of the field, which consists to retrieve a value for each point with coordinates $\mathrm{x}$ and $\mathrm{y}$ in the map. In (Gomez, Vaisman et al. 2010), the authors propose a multidimensional model handling fields. They define two types of fields, "field" and "tempfield" (spatial field and temporal field), and semantics for the operators associated to these data types. They include the notion of field dimensions and field measures. They define the "field dimension" as a dimension containing at least one level that is a field (temperature, precipitation...), the "field measure" as a measure represented by a field and the "field hierarchy" as a set of related field levels, which allows a field to be seen at different levels of granularity . They also propose a physical model for data warehouses with continuous fields. However, no implementation has been proposed and the hierarchical relationship between field levels has not been brought to light. (Bimonte and Myoung 2011) provide a multidimensional model that integrates field data independently from their implementation, as measures and dimensions. They also present a formal representation of the spatio-multidimensional model schema where they define the concepts of field dimensions, field measures, and field views. To our knowledge, no implementation including the continuous appearance of incomplete field or the multiresolution over incomplete fields has been proposed.

Representation of multidimensional data under different resolution levels or scales may be considered as multirepresentation. (Bernier, Bédard et al. 2005) proposes an approach to provide onDemand multi-scale maps. Although this approach models maps features at different scales by using spatial hierarchies, but it does not contain measures. (Yvan, Proulx et al. 2002) defines a UML-based conceptual model that integrates multiple geometric and semantic representations properties of spatial levels. However, this work does not present a complete multidimensional model with facts and hierarchies.

Moreover, (Bédard et al., 2002) suggests (without providing details) using a different spatial data warehouse for each representation. Therefore, changing the representation corresponds to move to another spatial data warehouse. (Gascueña and Guadalupe 2009) propose a conceptual model with a multi-representation of spatial members. They also 
propose a physical schema, but any implementation into a classical ROLAP architecture is presented. Finally, (McGuire, Gangopadhyay et al. 2008)define a snowflake schema for an environmental application where three dimensions represent the same spatial members at different resolutions.

\section{$7 \quad$ Conclusion and future work}

In this paper we present a multidimensional model for incomplete fields at several resolutions and its implementation in a SOLAP architecture based on standards (e.g. SQL and MDX). We are working on using spatial data mining to speed-up map algebra operations and implement a SOLAP visualization client. We also work in integrating other interpolation functions to generalize the proposed approach.

\section{Acknowledgements}

The authors wish to thank the FEDER and the region for funding this project and project partners who provide us the data.

\section{References}

Ahmed, T. O. and M. Miquel (2005). "Multidimensional Structures Dedicated to Continuous Spatiotemporal Phenomena." 01/2005; In proceeding of: Database: Enterprise, Skills and Innovation, 22nd British National Conference on Databases, BNCOD 22, Sunderland, UK, July 57, 2005, Proceedings.

Bédard, Y., T. Merrett, et al. (2001). "Fundamentals of Spatial Data Warehousing for Geographic Knowledge Discovery." Data Mining and Knowledge Discovery

Bédard, Y., S. Rivest, et al. (2007). "Spatial On-Line Analytical Processing (SOLAP): Concepts, Architectures and Solutions from a Geomatics Engineering Perspective." Data Warehouses and OLAP: Concepts, Architectures and Solutions. IGI Global, 2007. 298-319. Web. 23 Jan. 2013. doi:10.4018/987-1-59904-364-7.ch013.

Bernier, E., Y. Bédard, et al. (2005). "UMapIT: An OnDemand Web Mapping Tool Based On A Multiple Representation Database." $\underline{\text { 8th ICA }}$ WORKSHOP on Generalisation and Multiple Representation, A Coruña, July 7-8th.

Bimonte, S. and K. Myoung (2011). "Towards a Model for the Multidimensional Analysis of Field Data." Advances in Databases and Information Systems
,Lecture Notes in Computer Science Volume 6295, 2011, pp 58-72.

Camossi, E., E. Bertino, et al. (2009). " Adaptive Management of Multigranular Spatio-Temporal Object Attributes." SSTD 2009: 320-337.

Gascueña, C. M. and R. Guadalupe (2009). "A MULTIDIMENSIONAL METHODOLOGY WITH SUPPORT FOR SPATIO-TEMPORAL MULTIGRANULARITY IN THE CONCEPTUAL AND LOGICAL PHASES." IGI Global.

Gomez, L., A. Vaisman, et al. (2010). "Physical Design and Implementation of Spatial Data Warehouses Supporting Continuous Fields." DaWaK'10 Proceedings of the 12th international conference on Data warehousing and knowledge discovery, Pages 25-39

Gomez, L. I., S. A. Gomez, et al. (2012). "A Generic Data Model and Query Language for Spatiotemporal OLAP Cube Analysis." EDBT '12 Proceedings of the 15th International Conference on Extending Database Technology, Pages 300-311 ACM New York, NY, USA (02012

McGuire, M., A. Gangopadhyay, et al. (2008). "A usercentered design for a spatial data warehouse for data exploration in environmental research." Ecological Informatics, Volume 3, Issues 4-5, Pages 273-285.

McHugh, R. (2008). "Intégration de la structure matricielle dans les cubes spatiaux."

Mennis, J., R. Viger, et al. (2005). "Cubic Map Algebra functions for spatio-temporal analysis." Cartography and Geographic Information Systems.

Paolino, L., M. Sebillo, et al. (2010). "Integrating Discrete and Continuous Data in an OpenGeospatialCompliant Specification." Transactions in GIS Volume 14, Issue 6, pages 731-753, December $\underline{2010 .}$.

Parent, C., S. spaccapietra, et al. (2006). "Conceptual Modelling for Traditional and Spatio-temporal Applications." 2006 XVIII, 465 p. 115 Illus. Hardcover.

Yvan, B., M. J. Proulx, et al. (2002). "Modeling multirepresentations into spatial data warehouses : A UML-Based approach." Symposium on Geospatial Theory, Processing and Applications, Ottawa

O'Sullivan, D. and D. J.Unwin (2002). Geographic Information Analysis - John Wiley \& Sons, 15 nov. 2002 - 448 pages 
ICEIS 2013 INSTICC International Conference on Enterprise Information Systems, July 3-7, 2013, Angels 